\title{
Relações entre estratégias de coping e adaptabilidade acadêmica em estudantes universitários
}

\author{
Luana Luca ${ }^{1}$ \\ Ana Paula Porto Noronha \\ Francine Náthalie Ferraresi Rodrigues Queluz \\ Universidade São Francisco, Campinas-SP, Brasil
}

\section{Resumo}

O objetivo do presente estudo foi verificar se estudantes universitários com mais estratégias de coping em seu repertório se adaptam melhor ao ensino superior. Participaram do estudo 927 universitários com idade média de 26,04 anos $(D P=7,66)$, sendo 60,9\% do sexo feminino, que responderam ao Inventário de Estratégias de Coping (IEC) e ao Questionário de Adaptação ao Ensino Superior (QAES). Os resultados encontrados revelaram que as estratégias de coping, de maneira geral, se correlacionaram positivamente com a melhor adaptação ao ensino superior. Os dados encontrados nesta pesquisa podem servir para subsidiar futuras intervenções em universitários visando fortalecer suas estratégias de coping, e, por conseguinte, melhorar sua adaptação acadêmica.

Palavras-chave: Estratégias de enfrentamento, ensino superior, universitários, adaptabilidade

\section{Abstract: Relationships among coping strategies and academic adaptability in undergraduate students}

The objective of this research was to verify if undergraduate students with more coping strategies in their repertoire were better adapted to higher education. A total of 927 undergraduate students with a mean age of 26.04 years $(S D=7.66), 60.9 \%$ female, answered the Coping Strategies Inventory (IEC) and the Adaptation to Higher Education Questionnaire (QAES). The results showed that coping strategies, in general, correlated positively with a better adaptation to higher education. The data found in this study may serve to subsidize future interventions in undergraduate students aiming to strengthen their coping strategies, and therefore, to improve their academic adaptation.

Keywords: Coping strategies, higher education, university students, adaptability

\section{Resumen: Relaciones entre estrategias de coping y adaptabilidad académica en estudiantes universitarios}

El objetivo de esta investigación fue verificar si estudiantes universitarios con más estrategias de coping en su repertorio se adaptaron mejor a la enseñanza superior. En el estudio 927 universitarios con edad media de 26,04 años $(D P=7,66)$, siendo el 60,9\% del sexo femenino, respondieron al Inventario de Estrategias de Coping (IEC) y al Cuestionario de Adaptación a la Enseñanza Superior (QAES). Los resultados encontrados demostraron que las estrategias de coping, de manera general, se correlacionaron positivamente con una mejor adaptación a la enseñanza superior. Los datos encontrados en este estudio pueden servir para subsidiar futuras intervenciones en universitarios para fortalecer sus estrategias de coping, y por lo tanto, mejorar su adaptación académica.

Palabras clave: Estrategias de enfrentamiento, enseñanza superior, universitarios, adaptación

\footnotetext{
${ }^{1}$ Endereço para correspondência: Universidade São Francisco. Rua Waldemar Cesar da Silveira, 105, Jardim Cura D'ars, 13045-510, Campinas, SP. E-mail: luanaluca@gmail.com
} 
O conceito de coping se refere ao conjunto de estratégias que os indivíduos utilizam para controlar ou reduzir os efeitos estressantes em diferentes situações ao longo da vida. Essas estratégias são respostas expressas por meio dos pensamentos, sentimentos e ações que o sujeito desenvolve, sejam eles adaptados ou não para a interação com o ambiente (Lazarus \& Folkman, 1984). As estratégias de coping também estão associadas a resultados positivos e importantes para a promoção da saúde e do bem-estar. Este modo de lidar engloba crenças de que as situações se resolverão da melhor forma, por meio da responsabilização das consequências dos seus atos e comportamentos. Ou seja, este comportamento abrange uma visão positiva de sucesso, mas que é determinada pela capacidade do próprio indivíduo (Greenglass \& Fiksenbaum, 2009; Luhmann, Hofmann, Eid, \& Lucas, 2012).

Compreender o processo sobre como os indivíduos gerenciam diversas situações, embora seja uma preocupação existente há décadas, também se mostra atual. Existe uma prevalência de elementos adversos, e por isso uma grande necessidade de aumentar de forma positiva as capacidades humanas. As estratégias de coping desempenham um papel fundamental no bem-estar físico e psicológico, quando confrontados com desafios ou com momentos negativos. As estratégias que o sujeito utiliza podem ser direcionadas para a gestão de metas e para alcançar os seus objetivos. Por exemplo, ao utilizá-las na superação de dificuldades, estas podem contribuir também para a diminuição de níveis de estresse e melhor percepção de qualidade de vida (Akram \& Mahmood, 2013; Ferreira, Queluz, Ximenes, Isaac, \& Barham, 2017; Folkman \& Moskowitz, 2000; Greenglass, 2002). Percebe-se, então, que o coping tem um papel importante no ajustamento psicossocial e aponta para um maior compromisso com os objetivos de vida (Major, Holland, \& Oborn, 2012; Smedema, Catalano, \& Ebener, 2010).

Em uma perspectiva cognitivista, Folkman e Lazarus $(1980,1985)$ propuseram uma distinção entre dois tipos gerais de coping: aquele focado no problema e o focado na emoção. $\mathrm{O}$ coping focado no problema refere-se às tentativas, cognitivas e comportamentais, ativas, diretas e construtivas para modificar ou eliminar a situação geradora de estresse e, deste modo, resolver os problemas. O coping focado na emoção refere-se aos esforços para reduzir ou regular a resposta emocional que está associada ao evento estressor ou que resulta da situação estressante. Estas duas estratégias gerais de coping estão associadas a duas importantes funções do coping: a alteração da relação indivíduo-ambiente causadora de estresse e a regulação de emoções perturbadoras. De modo geral, os investigadores sugerem que o coping focado no problema é mais eficaz e provavelmente associado à saúde psicológica (Luhmann et al., 2012; Pinto \& Barham, 2014), enquanto as estratégias de coping emocionais são menos eficazes e estão associadas a maior perturbação psicológica (Luhmann et al., 2012; Pearlin \& Schooler, 1978).

$\mathrm{Na}$ literatura estrangeira, estudos têm investigado o coping no ambiente universitário (Douglass \& Duffy, 2015; Dyson \& Renk, 2006). Parte dessas pesquisas analisa as estratégias de enfrentamento e suas relações com o estresse (Galatzer-Levy, Burton, \& Bonanno, 2012), inteligência emocional (MacCann, Forgaty, Zeider, \& Roberts, 2011) e desempenho acadêmico (Collinsa \& Onwuegbuzie, 2003). No entanto, mesmo nesse contexto, não são frequentes as tentativas de relacionar o coping com a variável adaptação acadêmica. As publicações encontradas buscaram analisar quais são as estratégias de enfrentamento mais utilizadas e de que forma elas afetam o processo de adaptação acadêmica, mas não focaram nas possíveis relações entre os dois construtos (Douglass \& Duffy, 2015; Luhmann et al., 2012). No Brasil, Oliveira, Carlotto, Vasconcelos e Dias (2014) realizaram uma revisão da literatura na qual buscaram identificar possíveis relações entre adaptação acadêmica e estratégias de enfrentamento em estudantes universitários brasileiros. No entanto, concluíram que, dentre os estudos analisados, alguns tinham como objetivo construir e validar instrumentos, investigar vivências acadêmicas e descrever correlações entre coping e variáveis de saúde psicológica, mas nenhum abordou diretamente possíveis relações entre coping e adaptação acadêmica.

Diante disso, percebe-se que tem se tornado cada vez mais evidente a preocupação de pesquisadores em relação à integração e adaptação do estudante à vida universitária, mas ainda são necessários novos estudos visando entender as relações entre coping e adaptabilidade de carreira (Mognon \& Santos, 2014; Oliveira et al., 2014; Santos, Polydoro, Scortegagna, \& Linden, 2013; Soares, Baldez, \& Melo, 2011). Na atualidade, o ensino superior tem sido considerado como um dos fatores fundamentais para o desenvolvimento econômico, social e cultural das sociedades (Santos et al., 2013). A entrada e permanência no ensino superior implicam, muitas vezes, em mudanças marcantes na vida dos jovens, representando uma descontinuidade em relação a experiências pessoais, sociais e acadêmicas anteriores (Douglass \& Duffy, 2015).

Reason, Terenzini e Domingo (2007) enfatizaram que durante a graduação os alunos desenvolvem (ou deixam de desenvolver) atitudes, comportamentos, 
habilidades, conhecimentos e hábitos mentais, elementos dos quais dependerá sua preparação posterior e êxito acadêmico e profissional, além de ser também um período de intensa aprendizagem. Para Polydoro (2000), a entrada na universidade é uma transição importante para o indivíduo, pelo fato de ser um momento frequentemente sincronizado com as mudanças e adaptações peculiares da adolescência e vida adulta. De acordo com a autora, as pesquisas têm compreendido a adaptação ao ensino superior como um fenômeno multifacetado, tendo três grandes elementos que o influenciam: condições pessoais, características institucionais e grupos de interação.

Nesse sentido, esses elementos foram discutidos pelo modelo proposto por Tinto (1975), que identifica os fatores como relacionados com a persistência do aluno na universidade, referindo-se ao construto adaptação acadêmica. $\mathrm{O}$ primeiro, condições pessoais, inclui antecedentes familiares, habilidades e atributos desenvolvidos antes da vida universitária, e mais especificamente durante o ensino médio. $\mathrm{O}$ segundo fator está ligado às características institucionais, e refere-se ao campus no qual o aluno está matriculado. O terceiro, grupos de interação, diz respeito ao desempenho acadêmico do aluno e suas interações com professores e funcionários da instituição, relações entre os alunos e nas atividades extracurriculares. Para o autor, a adaptação/integração acadêmica ocorre quando o aluno passa a fazer parte da comunidade na qual está inserido e compartilha os valores comportamentais dos colegas e professores, as atitudes normativas, e respeita as exigências formais e informais. Nesse sentido, o ajustamento ao contexto universitário deve ser considerado como um processo construído no cotidiano das relações estabelecidas entre o aluno e a instituição.

$\mathrm{Na}$ mesma direção, Granado, Santos, Almeida, Soares e Guisande (2005) sustentam que o construto adaptação universitária é definido por dois componentes: por um lado, a troca entre as expectativas, habilidades e demais características dos estudantes; por outro lado, os componentes da universidade, isto é, sua comunidade, sua estrutura e elementos organizacionais. Sob essa perspectiva, entende-se a importância de avaliar o estudante, em qualquer que seja o curso, não apenas no que diz respeito às aulas, às atividades e avaliações curriculares, mas também é preciso considerar a maneira como o estudante encara e vive a experiência do ensino superior (Ambiel, Santos, \& Dalbosco, 2016; Oliveira et al., 2014).

Assim, o coping, objeto de estudo da presente pesquisa, pode ser considerado como uma das variáveis facilitadoras para um estado psicológico positivo e contribuir para a melhor adaptação acadêmica (Oliveira et al., 2014). As estratégias de coping são consideradas efetivas na redução das dificuldades encontradas em relação ao ambiente, da mesma forma que contribuem para o melhor ajuste do indivíduo aos problemas que encontra, tornando-se relevante a discussão do aspecto do ambiente, que essa produção será a respeito da adaptação acadêmica (Bédard, Bouffard, \& Pansu, 2014; Pinto \& Barham, 2014; Ramos, Enumo, \& Paula, 2015; Segrin, McNelis, \& Swiatkowski, 2016).

Desse modo, as possíveis relações entre coping e adaptação acadêmica serão investigadas, uma vez que, entende-se que determinadas estratégias de coping podem estar correlacionadas com uma melhor adaptação acadêmica. Uma adaptação acadêmica bem-sucedida implica, quer a adaptação e o envolvimento em tarefas acadêmicas e extracurriculares, quer o compromisso com a persistência (Ambiel et al., 2016; Oliveira et al., 2014), sendo que este nível de persistência é influenciado pela qualidade das redes formadas em contexto universitário (Bédard et al., 2014; Ramos et al., 2015).

Dado o exposto e diante da lacuna de conhecimento destacada por Oliveira et al. (2014) na literatura nacional, o objetivo desta pesquisa foi verificar se estudantes universitários com mais estratégias de coping em seu repertório se adaptaram melhor ao ensino superior. Como hipótese inicial, esperava-se que estudantes com um maior repertório em estratégias de coping apresentassem também melhor adaptação ao ensino superior, de forma que os construtos estivessem positivamente relacionados.

\section{Método}

\section{Participantes}

Participaram do presente estudo 927 estudantes universitários, com idade média de 26,04 anos $(D P=7,66)$, variando de 18 a 59 anos, sendo a maioria do sexo feminino $(60,6 \%)$ e solteiros $(68,3 \%)$. Os dados analisados constaram de participantes dos seguintes cursos de graduação, Psicologia (47,9\%), Engenharia $(36,4 \%)$ e Pedagogia $(15,8 \%)$ de uma instituição particular do interior de São Paulo (73\%) e outra do estado da Paraíba (27\%). Na Tabela 1 são apresentados os dados sociodemográficos dos participantes. Como critério de inclusão, os estudantes deveriam ter mais que 18 anos no momento da coleta de dados. 
Tabela 1

Perfil Sociodemográfico dos Participantes $(N=927)$

\begin{tabular}{lcc}
\hline \multicolumn{1}{c}{ Variável } & $\mathrm{n}$ & $\%$ \\
\hline Sexo & & \\
$\quad$ Feminino & 566 & 60,6 \\
$\quad$ Masculino & 361 & 39,4 \\
Estado Civil & & \\
$\quad$ Solteiro & 633 & 68,3 \\
$\quad$ Casado/Relação estável & 261 & 28,2 \\
$\quad$ Separado/Divorciado & 33 & 3,5 \\
Curso & & \\
$\quad$ Psicologia & 444 & 47,9 \\
$\quad$ Engenharia & 337 & 36,4 \\
Pedagogia & 146 & 15,8 \\
Instituição & & \\
$\quad$ Universidade particular (SP) & 677 & 73,0 \\
$\quad$ Universidade pública (PB) & 250 & 27,0 \\
\hline
\end{tabular}

\section{Instrumentos}

Inventário de Estratégias de Coping (Folkman \& Lazarus, 1985). Foi utilizada a versão brasileira do Inventário de Estratégias de Coping de Folkman e Lazarus (1984), adaptado para o português por Savóia, Santana e Mejias (1996) e com a estrutura fatorial sugerida por Luca (2017). O instrumento contém 50 itens que incluem pensamentos e ações utilizadas para lidar com demandas internas ou externas de qualquer evento estressor, que deve ser especificado pelo pesquisador antes da coleta. Nesse caso foi incluído no cabeçalho a orientação para que os participantes recorressem às dificuldades que vivenciaram no contexto acadêmico e que assim reportassem as estratégias utilizadas diante dessa realidade. Os itens são respondidos de acordo com a intensidade com que utilizam cada estratégia mencionada, em determinada situação, tendo como opções de respostas: (0) não usei esta estratégia, (1) usei um pouco, (2) usei bastante e (3) usei em grande quantidade. O Alfa de Cronbach geral do IEC encontrado por Luca (2017) foi de 0,95.

Questionário de Adaptação ao Ensino Superior (QAES) (Araújo et al., 2013). O QAES foi elaborado para avaliar a adaptação acadêmica de estudantes universitários em Portugal e adaptado para uso no Brasil. O instrumento consta de 40 itens, divididos em cinco dimensões, por meio da análise fatorial exploratória: Projeto de Carreira, Adaptação Social, Adaptação Pessoal-Emocional (os itens dessa dimensão devem ser invertidos), Adaptação ao Estudo e Adaptação Institucional. Os índices de consistência interna dos itens em cada uma das dimensões são satisfatórios, sendo o menor $\alpha=0,72$ para Adaptação Institucional e o maior para Adaptação Pessoal-Emocional $(\alpha=0,92)$.

\section{Procedimentos de coleta de dados}

Primeiramente foi solicitada autorização das instituições participantes e posteriormente foi realizado o envio do projeto para o Comitê de Ética da Universidade São Francisco, o qual foi aprovado com Protocolo CAAE 50003715.7.0000.5514. Após a aprovação, os estudantes foram convidados a participar do presente estudo nas universidades na qual estudavam e após o aceite, assinaram o Termo de Consentimento Livre Esclarecido (TCLE), tendo ciência dos propósitos do estudo e das questões éticas envolvidas. Foram informados dos objetivos do estudo e solicitados para que respondessem aos dois instrumentos. As aplicações foram coletivas, em sala de aula, com duração aproximada de 30 minutos.

\section{Procedimentos de análise de dados}

Para verificar a distribuição dos escores para cada instrumento, foram calculados a média, o desvio padrão, os valores mínimos e máximos, além de indicadores de curtose e simetria para cada variável. Todas as variáveis apresentaram uma distribuição normal, segundo a inspeção do número de modas, valores de curtose e assimetria e do teste de normalidade de Kolmogorov-Smirnov (Marôco, 2014). Assim, foi utilizado o coeficiente de correlação de Pearson para verificar possíveis correlações entre as variáveis. Para o presente estudo, a magnitude das correlações foi classificada em: Fraca $(<0,3)$; Moderada $(0,3$ a 0,59$)$, Forte $(0,60$ a 0,99$)$ ou Perfeita $(1,0)$ (Levin $\&$ Fox, 2004). Os dados foram analisados com o software SPSS Statistics 20.

\section{Resultados e discussão}

Nos resultados apresentados na Tabela 2, pode-se observar que a maioria dos construtos do IEC e do QAES foram estatisticamente correlacionados, mostrando que, de maneira geral, a adaptação ao ensino superior está correlacionada com a maior presença de estratégias de coping no repertório de universitários. A maioria das correlações foi de magnitude fraca e as únicas que apresentaram magnitude moderada foram entre o fator Adaptação pessoal emocional do QAES com os fatores Afastamento e Aceitação e Reavaliação positiva do IEC. 
No que diz respeito ao escore total do QAES, o único fator do IEC que não teve correlação estatisticamente significativa foi o fator Afastamento e aceitação. O escore total do IEC se correlacionou positivamente com todas as medidas do QAES, com correlações de magnitude fraca. No fator Afastamento e aceitação do IEC, 4 entre 6 fatores apresentaram correlação negativa com as medidas do QAES.

Tabela 2

Correlações entre Escores do Inventário de Estratégias de Coping (IEC) e Escores no Questionário de Adaptação ao Ensino Superior

\begin{tabular}{lccccc}
\hline & IEC Total & $\begin{array}{c}\mathrm{F} 1 \\
\text { Resolução de } \\
\text { problemas }\end{array}$ & $\begin{array}{c}\mathrm{F} 2 \\
\text { Afastamento } \\
\text { e aceitação }\end{array}$ & $\begin{array}{c}\mathrm{F} 3 \\
\text { Reavaliação } \\
\text { Positiva }\end{array}$ & $\begin{array}{c}\text { F4 } \\
\text { Suporte } \\
\text { Social }\end{array}$ \\
\hline QAES Total & $0,24^{* *}$ & $0,30^{* *}$ & 0,02 & $0,15^{* *}$ & $0,25^{* *}$ \\
QAES - F1 - Projeto de carreira & $0,12^{* *}$ & $0,26^{* *}$ & $-0.13^{* *}$ & 0,02 & $0,17^{* *}$ \\
QAES - F2 - Adaptação social & $0,13^{* *}$ & $0,25^{* *}$ & $-0,11^{* *}$ & $-0,04$ & $0,19^{* *}$ \\
QAES - F3 - Adaptação pessoal emocional & $0,18^{* *}$ & 0,05 & $0,40^{* *}$ & $0,32^{* *}$ & 0,04 \\
QAES - F4 - Adaptação ao estudo & $0,09^{* *}$ & $0,26^{* *}$ & $-0,18^{* *}$ & $-0,05$ & $0,15^{* *}$ \\
QAES - F5 - Adaptação institucional & $0,07^{*}$ & $0,13^{* *}$ & $-0,05$ & $-0,10$ & $0,12^{*}$ \\
\hline
\end{tabular}

$* p<.05, * * p<.01$

O objetivo desta pesquisa foi verificar se estudantes com mais estratégias de coping em seu repertório se adaptaram melhor ao ensino superior. Diante disso, tem-se confirmada a hipótese inicial, uma vez que os construtos em seus escores gerais se mostraram estatisticamente correlacionados, ou seja, estudantes universitários com um maior repertório de estratégias de coping se adaptam melhor ao entrar no mundo acadêmico. Esse dado também se confirma, uma vez que todos os fatores do QAES se correlacionaram com o escore total do IEC, mostrando que as estratégias de coping podem ser fundamentais para que os estudantes consigam se adaptar à vida universitária (Galatzer-Levy et al., 2012; Oliveira et al., 2014; Santos et al., 2013). No que diz respeito ao escore total do QAES, o único fator do IEC que não se mostrou correlacionado foi o fator Afastamento e Aceitação. Diante disso, levanta-se a hipótese de que principalmente o comportamento de afastar-se pode não ser adaptativo para o estudante ao se inserir no ensino superior, uma vez que ao se comportar dessa maneira, o mesmo pode não ter acesso a situações de aprendizagem que seriam essenciais para o sucesso acadêmico (Santos et al., 2013).

No que diz respeito especificamente aos fatores, percebe-se que o mesmo fator Afastamento e Aceitação do IEC apresentou correlações negativas com a maioria dos fatores do QAES, diferente dos outros que apresentaram correlações positivas. Uma possível hipótese para esse resultado é o fato de que estudantes que optam por afastar-se de determinadas situações podem não conseguir se adaptar a determinadas situações que o ensino superior demanda, uma vez que inserir-se em novos contextos pode ser essencial para uma melhor inserção e aprendizagem (como já dito) e especificamente no que diz respeito à adaptabilidade ao estudo, no quesito social e de carreira (Ambiel et al., 2016; Santos et al., 2013). No entanto, esse mesmo fator se correlacionou positivamente com o fator Adaptação pessoal emocional do QAES, mostrando que se afastar e aceitar as situações pode favorecer a adaptação pessoal e emocional no ensino superior, o que também faz sentido teórico, uma vez que o estudante pode se afastar de situações das quais não se sente confortável, mas passa a aceitar situações do dia a dia na universidade que tem que enfrentar e não conseguiria mudar com o afastamento (Major et al., 2012), o que contribui para sua adaptação pessoal e emocional.

O fator Resolução de problemas do IEC se correlacionou positivamente com todos os fatores do QAES, exceto o fator Adaptação emocional pessoal. Isso indica que um estudante conseguir se organizar para resolver os problemas pode ajudá-lo em questões práticas do dia a dia, como, por exemplo, as relacionadas à instituição ou ao estudo em si, mas que outro tipo de habilidades, como as voltadas para a emoção, podem ser fundamentais para o desenvolvimento de questões que envolvem fatores emocionais e pessoais, por exemplo. Esses dados corroboram os estudos de Lazarus e Folkman (1985) ao dividirem as estratégias de coping em voltadas para a emoção ou para a resolução de problemas e ao sugerir que as últimas estão associadas a uma melhor sensação de bem-estar psicológico (Luhmann et al., 2012; Pinto \& Barham, 2014). O fator Reavaliação positiva do IEC foi o fator que menos se correlacionou com as medidas avaliadas no QAES, tendo se correlacionado somente com 
Projeto de carreira e Adaptação pessoal emocional. Ambas correlações foram positivas e podem indicar que uma melhor avaliação e reavaliação do universitário em relação a si mesmo, pode ter influência positiva em seus projetos de carreira, pois, uma vez que se avalia de forma mais positiva, se sente mais confiante com a carreira que escolheu cursar no ensino superior, o que faz com que se sinta melhor e, consequentemente, se adapte melhor também pessoal e emocionalmente (Ambiel et al., 2016; Douglass \& Duffy, 2015).

Por fim, o fator Suporte social do IEC se correlacionou positivamente com quase todos os fatores do QAES, exceto com o fator Adaptação emocional pessoal. Esse dado foi inesperado uma vez que há estudos (Bédard et al., 2014; Segrin et al., 2016) que mostraram que o suporte social se relacionou com uma melhor adaptação emocional e pessoal em diferentes áreas da vida. Uma hipótese para esse resultado é que talvez a adaptação emocional e pessoal seja mais influenciada por fatores externos ao ambiente acadêmico; esses fatores podem se relacionar, por exemplo, com a saúde mental atual do estudante ou até com a sua certeza sobre a carreira escolhida (Ambiel et al., 2016), o que influenciaria sua Adaptação emocional pessoal, mas não o Suporte social percebido.

Dado o exposto, percebe-se que estudar e entender a forma como as variáveis adaptação ao ensino superior e estratégias de coping se relacionam é de extrema importância, uma vez que, ao analisar os construtos envolvidos nesse contexto, pode-se pensar também na realização de intervenções em universitários em anos iniciais de estudo visando facilitar sua adaptação ao ensino superior. Por exemplo, ao desenvolver suas estratégias de resolução de problemas ou trabalhar com o estudante como obter suporte social, espera-se que o mesmo consiga se adaptar a diferentes áreas no contexto acadêmico, como carreira, social, estudo e institucional. Ao focar no desenvolvimento de estratégias que tenham como foco todas as estratégias de coping, espera-se que, ao mesmo tempo, o estudante se adapte melhor a todas as áreas que envolvem o ensino superior.

\section{Considerações finais}

O presente estudo pretendeu discutir as relações entre estratégias de coping e adaptação acadêmica. Acredita-se que ele contribuiu com os achados da área, pois poderá subsidiar intervenções que tenham foco no desenvolvimento de estratégias de coping em universitários, assim como para um melhor entendimento de como essas variáveis se relacionam, visando ao desenvolvimento de estratégias específicas, a depender do foco da intervenção. Ainda no que se refere às relações encontradas, por meio delas é possível aprimorar a qualidade dos instrumentos psicológicos, colocando-os à prova e confirmando ou rejeitando suas concepções teóricas.

Como limitação, tem-se o fato de que os dados foram coletados nos estados de São Paulo e da Paraíba. Se os dados tivessem sido coletados em outras regiões ou estados, eles poderiam ser generalizados para o Brasil, mas considerando as medidas continentais do pais, é necessário ter cautela. Em estudos futuros seria importante ampliar a amostra para outras regiões para verificar se os resultados se manteriam, bem como realizar estudos com delineamentos quase-experimentais, com aplicação de pré e pós teste, para verificar se haveria mudança na adaptação ao ensino superior após uma intervenção focada no desenvolvimento de estratégias de coping. Ainda a título de agenda de pesquisa, seria possível verificar as relações entre adaptabilidade acadêmica e estratégias de coping em estudantes com diferentes escolaridades, a fim de identificar se as estratégias de coping se relacionam com maior escolaridade.

\section{Referências}

Akram, F., \& Mahmood, K. (2013). Gender Differences in Coping Strategies and Life Satisfaction Among Cardiac Patients. Institute of Interdisciplinary Business Research, 5(5), 537-552.

Ambiel, R.A. M., Santos, A. A. A., \& Dalbosco, S. N. P. (2016). Motivos para evasão, vivências acadêmicas e adaptabilidade de carreira em universitários. Psico, 47(4), 288-297. doi: 10.15448/1980-8623.2016.4.23872

Araújo, A. M., Almeida, L. S., Ferreira, J. A., Santos, A. A., Noronha, A. P., \& Zanon, C. (2014). Questionário de Adaptação ao Ensino Superior (QAES): Construção e validação de um novo questionário. Psicologia, Educação e Cultura, 18(1), 131-145.

Bédard, K., Bouffard, T., \& Pansu, P. (2014). The risks for adolescents of negatively biased self-evaluations of social competence: The mediating role of social support. Journal of adolescence, 37(6), 787-798. doi: 10.1016/j. adolescence.2014.05.004

Collinsa, K. M. T., \& Onwuegbuzie, A. J. (2003). Study coping and examination-taking coping strategies: The role of learning modalities among female graduate students. Personality and Individual Differences, 35(5) 1021-1032. 
Douglass, R. P., \& Duffy, R. D. (2015). Calling and career adaptability among undergraduate students. Journal of Vocational Behavior, 86(2015), 58-65. doi: 10.1016/j.jvb.2014.11.003

Dyson, R., \& Renk K. (2006). Freshmen adaptation to university life: Depressive symptoms, stress, and coping. Journal of Clinical Psychology, 62(10), 1231-1244.

Ferreira, C. R., Queluz, F. N. F. R., Ximenes, V. S., Isaac, L., \& Barham, E. (2017). P3Es e a diminuição da sobrecarga em cuidadores: Confirmando efeitos em curto e longo prazo. Revista Kairós - Gerontologia, 20(3), 131-150. doi: $10.23925 / 2176-901 X .2017 \mathrm{v} 20 \mathrm{i} 3 \mathrm{p} 131-150$

Folkman, S., \& Lazarus, R. S. (1980). An analysis of coping in a middle-aged community sample. Journal of Health and Social Behavior, 21(3), 219-239.

Folkman, S., \& Lazarus, R. S. (1985). If it changes it must be a process: Study of emotion and coping during three stages of a college examination. Journal of Personality and Social Psychology, 48(1), 150. doi: 10.1037/0022-3514.48.1.150

Folkman, S., \& Moskowitz, J. T. (2000). Positive Affect and the Other Side of Coping. American Psychologist, 55, $647-654$

Galatzer-Levy, I. R., Burton, C. L., \& Bonanno, G. A. (2012). Coping flexibility, potentially traumatic life events, and resilience: A prospective study of college student adjustment. Journal of Social and Clinical Psychology, 31(6), 542-567.

Granado, J. I. F., Santos, A. A., Almeida, L. S., Soares, A. P., \& Guisande, M. A. (2005). Integração acadêmica de estudantes universitários: Contributos para a adaptação e validação do QVA-r no Brasil. Psicologia e Educação, IV(1), 33-43.

Greenglass, E. (2002). Chapter 3. Proactive Coping. In E. Frydenberg (Coord.), Beyond Coping: Meeting Goals, Vision, and Challenges (pp.37-62). London: Oxford University Press.

Greenglass, E.R., \& Fiksenbaum, L. (2009). Proactive Coping, Positive Affect and Well-Being: Testing for Mediation Using Path Analysis. European Psychologist, 14(1), 29-39. doi 10.1027/1016-9040.14.1.29.

Lazarus, R. S., \& Folkman, S. (1984). Stress, appraisal, and coping. New York: Springer.

Levin, J., \& Fox, J. A. (2004). Estatística para ciências humanas. São Paulo, SP: Pearson.

Luca, L. (2017). Estudos psicométricos do inventário de coping: Relações com forças de caráter e adaptação acadêmica. Tese de doutorado em Psicologia, Universidade São Francisco.

Luhmann, M., Hofmann, W., Eid, M., \& Lucas, R. E. (2012). Subjective Well-Being and Adaptation to Life Events: A Meta-Analysis. Journal of Personality and Social Psychology, 102(3), 592-615. doi:10.1037/a0025948.

MacCann, C., Forgarty, G., Zeidner, M., \& Roberts, R. (2011). Coping mediates the relationship between emotional intelligence (EI) and academic achievement. Contemporary Educational Psychology, 36(1), 60-70.

Major, D., Holland, J., \& Oborn, K. (2012). The Influence of Proactive Personality and Coping on Commitment to STEM Majors. The Career Development Quarterly, 60(1), 16-24. doi: 10.1002/j.2161-0045.2012.00002.x.

Marôco, J. (2014). Análise estatística com o SPSS Statistics. Pêro Pinheiro, Portugal: Report Number

Mognon, J. F., \& Santos, A. A. A. (2014). Autoeficácia para dirigir, desengajamento moral e impulsividade em motoristas. Psico-USF, 19(3), 457-466. doi: 10.1590/1413-82712014019003008

Oliveira, C. T., Carlotto, R. C., Vasconcelos, S. J. L., \& Dias, A. C. G. (2014). Adaptação Acadêmica e Coping em Estudantes Universitários Brasileiros: Uma Revisão de Literatura. Revista Brasileira de Orientação Profissional, 15(2), 177-186.

Pearlin, L. I, \& Schooler, C. (1978). The structure of coping. Journal of Health and Social Behavior, 19(1), 2-21.

Pinto, F. N. F. R., \& Barham, E. J. (2014). Habilidades sociais e estratégias de enfrentamento de estresse: Relação com indicadores de bem-estar psicológico em cuidadores de idosos de alta dependência. Revista Brasileira de Geriatria e Gerontologia, 15(3), 525-539. doi: 10.1590/1809- 9823.2014.13043

Polydoro, S. A. J. (2000). O trancamento de matrícula na trajetória acadêmica do universitário: condições de saída e de retorno à instituição. Tese de Doutorado, Universidade de Campinas.

Ramos, F. P., Enumo, S. R. F., \& Paula, K. M. P. (2015). Teoria Motivacional do Coping: Uma proposta desenvolvimentista de análise do enfrentamento do estresse. Estudos de Psicologia (Campinas), 32(2), 269-279. doi: 10.1590/0103-166X2015000200011

Reason, R. D., Terenzini, P. T., \& Domingo, R. J. (2007). Developing social and personal competence in the first year of college. The Review of Higher Education, 30(3), 271-299. doi: 10.1353/rhe.2007.0012

Santos, A. A. A., Polydoro, S. A. J., Scortegagna, S. A., \& Linden, M. S. S. (2013). Integração ao ensino superior e satisfação acadêmica em universitários. Psicologia: Ciência e Profissão, 33(4), 780-793. https://dx.doi.org/10.1590/ S1414-98932013000400002 
Savóia, M. G., Santana, P. R., \& Mejias, N. P. (1996). Adaptação do Inventário de Estratégias de Coping de Folkman e Lazarus para o português. Psicologia USP, 7(1), 183-201. Recuperado de http://pepsic.bvsalud.org/pdf/psicousp/ v7n1-2/a09v7n12.pdf

Segrin, C., McNelis, M., \& Swiatkowski, P. (2016). Social skills, social support, and psychological distress: A test of the Social Skills Deficit Vulnerability Model. Human Communication Research, 42(1), 122-137. doi: 10.1111/ hcre. 12070

Smedema, S. M.., Catalano, D., \& Ebener, D. J. (2010). The Relationship of Coping, Self-Worth, and Subjective Well-Being. A Structural Equation Model, Rehabilitation Counseling Bulletin, 53(3),131-142. doi:10.1177/003455209358272.

Soares, A. B., Baldez, M. O. M., \& Mello, T. V. S. (2011). Vivências acadêmicas em estudantes universitários do estado do Rio de Janeiro. Interação em Psicologia, 15(1), 59-69.

Tinto, V. (1975). Dropout from higher education: A theoretical synthesis of recent research. Review of educational research, 45(1), 89-125.

Recebido 15/02/2018

$1^{a}$ Reformulação 05/09/2018

Aceite Final 19/10/2018

Sobre as autoras

Luana Luca é Doutora em Psicologia pelo Programa de Pós-Graduação Stricto Sensu em Psicologia da Universidade São Francisco, Campinas-SP.

Ana Paula Porto Noronha é Doutora em Psicologia Ciência e Profissão pela Pontifícia Universidade Católica de Campinas. Docente do Programa de Pós-Graduação Stricto Sensu em Psicologia da Universidade São Francisco, Campinas-SP. Bolsista Produtividade em Pesquisa do CNPq.

Francine Náthalie Ferraresi Rodrigues Queluz é Doutora em Psicologia pela Universidade Federal de São Carlos e Pós-doutoranda também em Psicologia pela Universidade São Francisco, Campinas-SP. 